\title{
Compósitos de Polietileno Reciclado e Partículas de Madeira de Reflorestamento Tratadas com Polietileno Modificado
}

\author{
Karla I. Redighieri, Dilma A. Costa \\ Departamento de Engenharia Química, IT, UFRRJ
}

\begin{abstract}
Resumo: Neste trabalho foram obtidos compósitos poliméricos de partículas de madeira de eucalipto e polietileno de baixa densidade reciclado (LDPE reciclado). O polietileno funcionalizado com anidrido maleico (PE-g-MA) foi utilizado como agente compatibilizante para conferir melhor adesão entre a matriz e a fase dispersa. As propriedades mecânicas de tração e flexão foram determinadas de acordo com as normas da American Society for Testing and Materials (ASTM). Os resultados mostraram que o tratamento das partículas de eucalipto com PE-g-MA aumentou as propriedades mecânicas dos compósitos, em relação aos compósitos contendo partículas sem tratamento. Entretanto, as propriedades mecânicas de tração desses compósitos foram reduzidas depois de longo tempo de imersão em água.
\end{abstract}

Palavras-chave: Compósitos, agentes compatibilizantes, propriedades mecânicas.

\section{Composites of Recycled Polyethylene and Reforestation Wood Particles Treated With Modified Polyethylene}

Abstract: In this work polymeric composites were obtained from eucalypt wood particles and recycled low density polyethylene (recycled LDPE). The maleic anhydride functionalized PE (PE-g-MA) was used as compatibiliser agent to provide better adhesion between the matrix and the dispersed phase. The mechanical properties of tensile and flexural were determined in accordance with the American Society for Testing and Materials (ASTM) procedures. The results showed that PE-g-MA as compatibiliser agent increased the mechanical properties, in relation to the composites containing particles without treatment. However, the tensile mechanical properties of these polymeric composites were reduced after long time of immersion in water.

Keywords: Composites, compatibiliser agent, mechanical properties.

\section{Introdução}

Os polímeros sintéticos e os naturais modificados têm contribuído muito para a poluição ambiental, principalmente quando se refere aos danos causados pelos resíduos urbanos ${ }^{[1]}$. Desse modo, ao invés de se desenvolver um novo polímero, que pode ou não apresentar as propriedades desejadas, pesquisas e desenvolvimento vêm sendo direcionadas para a modificação de polímeros já existentes, resultando em compósitos poliméricos, através da incorporação de cargas $(\text { fillers })^{[2,3]}$.

A produção de compósitos de plástico-madeira ou WPC (wood plastic composites) já é conhecida de muitos anos. Historicamente, a maioria desses compósitos usa pó de madeira como carga para plásticos. O pó de madeira diminui o custo, mas não necessariamente melhora o desempenho desses materiais. Mais recentemente, o uso de fibras naturais para promover um caráter de reforço para materiais termoplásticos tem sido de substancial interesse ${ }^{[4,5]}$.

A principal preocupação em se obter reforços verdadeiros através da incorporação de fibras de madeira, está na inerente incompatibilidade entre as fibras hidrofílicas e os polímeros hidrofóbicos, a qual resulta em uma pobre adesão, e consequentemente em uma fraca habilidade em transferir esforço da matriz para a fibra reforçante ${ }^{[4,5]}$.
A preparação desses compósitos poliméricos com madeira requer uma boa compatibilização na interface da matriz polimérica com a madeira ${ }^{[6]}$. Assim, alguns agentes compatibilizantes têm sido utilizados na modificação de fibras vegetais, para aumentar essa adesão interfacial entre o reforço celulósico e a matriz polimérica poliolefínica e com isso melhorar as propriedades mecânicas do compósito polimérico ${ }^{[7-12]}$.

Esse agente compatibilizante ou agente de acoplamento deve interagir fortemente com as fibras através de ligações covalentes ou fortes interações secundárias do tipo ácido-base ou ligações de hidrogênio. Isso implica que uma quantidade suficiente de grupos funcionais deve estar presente no compatibilizante, possibilitando a reação com as hidroxilas da celulose. Outro aspecto considerado é o comprimento das cadeias do compatibilizante que deve possuir cadeias suficientemente longas de alta massa molecular que permita a formação de emaranhados moleculares com a matriz polimérica, na interface, através de ancoramento mecânico ${ }^{[6,10-12]}$.

Normalmente, o tipo de madeira mais utilizado na confecção de aglomerados, compensados e chapas é a madeira de pinnus. No entanto, é cada vez mais premente a necessidade de se buscar novas alternativas de madeira para abastecimento das indústrias do setor ${ }^{[13]}$. 
Diante dessa demanda crescente pela madeira de pinnus surge a utilização de espécies alternativas de reflorestamento, tais como inúmeras espécies de eucalipto disponíveis em grandes áreas de plantios comerciais e experimentais. Além disso, uma motivação para a obtenção de compósitos de fibra natural e materiais termoplásticos é dar maior valor agregado a plásticos reciclados ${ }^{[14,5]}$.

Nesse sentido, este trabalho teve como objetivo desenvolver novos materiais compósitos poliméricos, que apresentem propriedades específicas, a partir da utilização de polietileno residual e madeira de reflorestamento (eucalipto) tratada com PE-g-MA, visando diminuir o impacto ambiental e agregar valor a esse novo material.

\section{Materiais e Métodos}

Os materiais empregados foram: LDPE reciclado $(\mathrm{MFI}=3,7008 \mathrm{~g} / 10 \mathrm{~min}$ ) fornecido pela Plasmar Ltda; PE-g-MA (MFI = 2,4192 g/10 min) fornecido pela Cromptom LTDA e partículas de madeira de eucalipto, de granulometria entre $2,03-0,64 \mathrm{~mm}$ e de densidade igual a $0,53 \mathrm{~g} / \mathrm{cm}^{3}$, fornecida pelo DPF/IF/UFRRJ.

As partículas de eucalipto foram tratadas com PE-g-MA, em duas diferentes concentrações, em relação ao peso das partículas, usando xileno como solvente. Após 10 minutos de imersão, as partículas de eucalipto tratadas sofreram filtração e foram levadas à estufa para secagem até peso constante. Para o cálculo do teor de PE-g-MA incorporado nessas partículas, foi feita a extração do PE-g-MA, em xileno, por 2 horas e secagem das partículas de eucalipto até peso constante. O teor de PE-g-MA incorporado foi determinado pela diferença de massa das amostras, antes e após a extração do PE-g-MA, tomando como referência o processo de extração realizado nas partículas de madeira sem tratamento, seguindo o mesmo procedimento.

Os compósitos de partículas de eucalipto (PEuc) sem tratamento e tratadas com o agente compatibilizante (PE-g-MA), nas duas concentrações, e polietileno reciclado (LDPErec) foram preparados em três composições distintas: 40/60, 50/50 e 60/40\%, em massa, de PEuc/LDPErec.

A preparação desses compósitos foi efetuada em plastógrafo Haake Rheocord System 9000, acoplado a câmara de mistura Rheomix 600, com rotor do tipo Roller, durante 10 minutos, a $150{ }^{\circ} \mathrm{C}$ e rotação de $40 \mathrm{rpm}$ e foram prensados em prensa hidráulica Freed S. Carver, modelo C, aquecida a $150{ }^{\circ} \mathrm{C}$, com carga de 8 toneladas, por 10 minutos.

Esses compósitos foram analisados termicamente a partir da técnica de termogravimetria (TG). A morfologia desses compósitos foi analisada através da microscopia eletrônica de varredura (SEM) e as propriedades mecânicas foram determinadas realizando ensaios de tração e flexão.

$\mathrm{Na}$ análise de TG as amostras foram aquecidas desde 30 à $700{ }^{\circ} \mathrm{C}$, numa razão de $20{ }^{\circ} \mathrm{C} / \mathrm{min}$, durante 40 minutos, em atmosfera de nitrogênio. As temperaturas iniciais de de- gradação térmica foram determinadas através das curvas de perda de massa com variação de temperatura.

A análise de SEM foi realizada em microscópio eletrônico de varredura marca JEOL modelo JSM-5300. As amostras dos compósitos foram metalizadas com ouro e analisadas com voltagem de aceleração de elétrons de $10 \mathrm{kV}$ e magnitude de 100.

As propriedades mecânicas dos compósitos foram determinadas mediante ensaio de tração (norma ASTM D 638-03) e ensaio de flexão (norma ASTM D 790-03) $)^{[15,16]}$.

Também foram realizados estudos de absorção de água dos compósitos. Os corpos de prova foram cortados nas mesmas dimensões da norma ASTM D 638-03 e foram imersos em água destilada, à temperatura ambiente. $\mathrm{O}$ aumento do peso foi medido periodicamente durante os 20 dias de imersão.

\section{Resultados e Discussão}

\section{Teor de PE-g-MA incorporado na superfície das partículas de madeira}

As partículas de madeira de eucalipto tratadas com PE-g-MA, nas duas concentrações, em relação ao peso das partículas, obtiveram graus de incorporação de 4,1 e $8,8 \%$, em massa.

\section{Compósitos de polietileno reciclado e madeira de reflorestamento}

Os compósitos poliméricos de partículas de madeira de eucalipto/polietileno de baixa densidade reciclado (PEuc/LDPErec), nas diferentes composições, mostraram-se bastante compactos e de fácil manuseio. As Figuras 1 e 2 apresentam as fotos das partículas de madeira de eucalipto tratadas com PE-g-MA e da placa produzida após prensagem do material compósito obtido no plastógrafo Haake.

\section{Análise termogravimétrica (TG)}

O objetivo da análise termogravimétrica (TG) foi determinar a temperatura inicial de degradação (Tonset) dos homopolímeros (LDPErec e PE-g-MA), além de verificar também a temperatura inicial de degradação (Tonset ou TG) dos compósitos obtidos, nas diferentes composições.

As curvas termogravimétricas obtidas para o LDPErec e o PE-g-MA estão apresentadas na Figura 3.

Os polímeros, LDPErec e PE-g-MA, apresentaram os seguintes valores de temperatura inicial de degradação, 225 e $298^{\circ} \mathrm{C}$, respectivamente.

As curvas termogravimétricas dos compósitos de PEuc/LDPErec apresentaram 02 temperaturas de degradação distintas, como mostrado nas Figuras 4-6. Essas curvas termogravimétricas apresentaram ligeira perda de massa em torno de $120^{\circ} \mathrm{C}$, atribuída a perda de umidade por parte das partículas de eucalipto. Foram identificados dois intervalos de perda de massa bastante significativos. Esses intervalos 


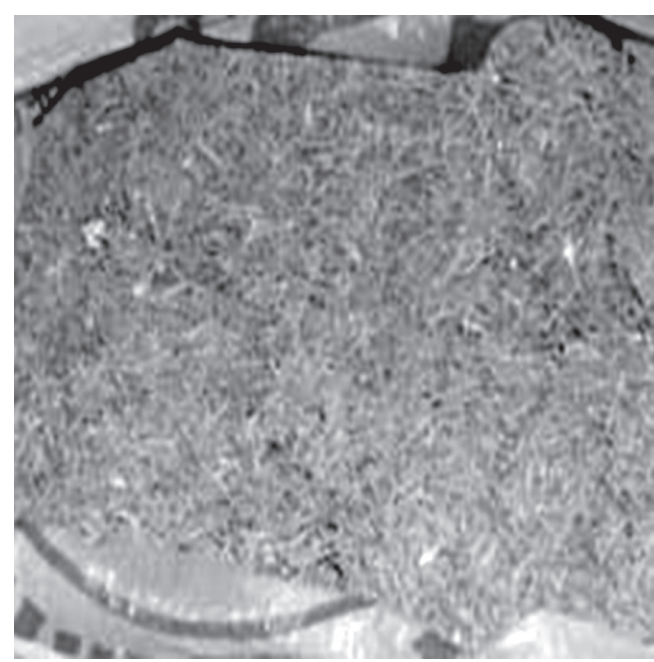

Figura 1. Partículas de madeira de eucalipto tratadas com PE-g-MA.

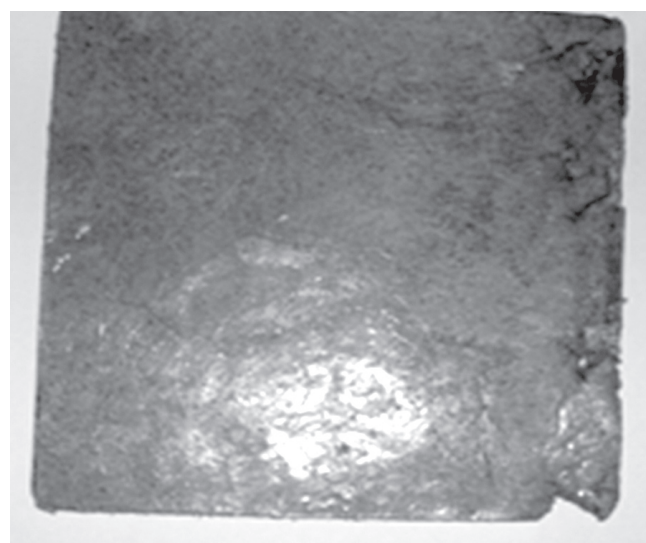

Figura 2. Placa prensada do compósito polimérico de PEuc/LDPErec $(40 / 60 \% \mathrm{~m} / \mathrm{m})$ obtida na prensa hidráulica.

foram atribuídos as fases contendo maiores proporções de partículas de madeira $\left(\mathrm{TG}^{1}\right)$ e maiores proporções de LDPErec $\left(\mathrm{TG}^{2}\right)$, respectivamente.

A Tabela 1 apresenta os valores de temperatura inicial de degradação $\left(\mathrm{TG}^{1}\right.$ e $\left.\mathrm{TG}^{2}\right)$ dos compósitos PEuc/LDPErec contendo partículas sem tratamento e tratadas com PE-g-MA.

Os compósitos de PEuc/LDPErec com partículas de madeira sem tratamento mostraram que a temperatura inicial de degradação $\left(\mathrm{TG}^{1}\right)$, atribuída a fase com maior proporção de partículas de madeira de eucalipto, diminuiu de valor com o aumento da proporção de madeira. Porém a composição de maior proporção de madeira (60/40\%) apresentou uma temperatura inicial de degradação $\left(\mathrm{TG}^{1}\right)$ superior à composição $(50 / 50 \%)$. Nesse caso, as partículas de madeira podem estar atuando como reforço para o LDPErec, aumentando ligeiramente a estabilidade térmica da fase de partículas de madeira. Essa indicação de reforço pode ser comprovada pelos valores de temperatura inicial de degradação da fase rica em LDPE reciclado $\left(\mathrm{TG}^{2}\right)$, que se apresentaram superiores ao valor da temperatura inicial de degradação do LDPErec puro, para todas as composições.

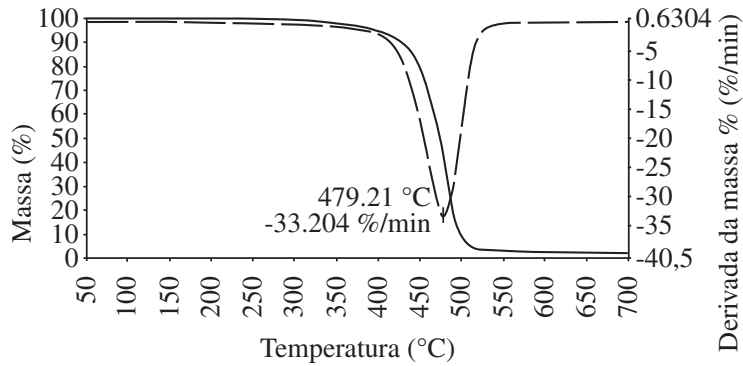

(a)

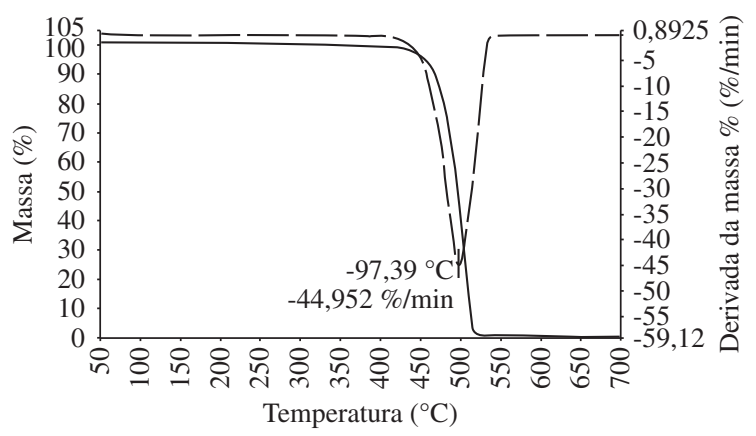

(b)

Figura 3. Curvas termogravimétricas dos homopolímeros: a) LDPErec; e b) PE-g-MA.

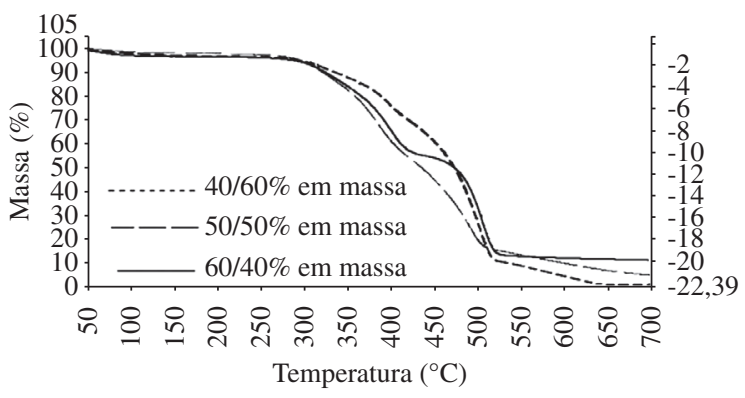

Figura 4. Curvas termogravimétricas dos compósitos PEuc/LDPErec com partículas de madeira de eucalipto sem tratamento, nas composições de $40 / 60 \%$; 50/50 e 60/40\% em massa, analisadas a $20{ }^{\circ} \mathrm{C} / \mathrm{min}$, sob atmosfera inerte de nitrogênio.

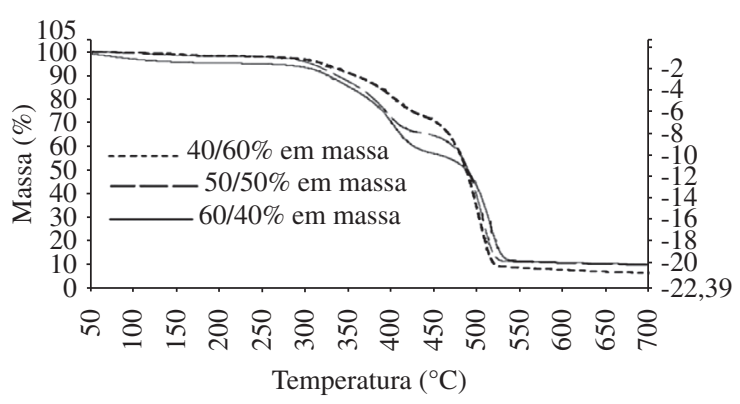

Figura 5. Curvas termogravimétricas dos compósitos (PEuc/LDPErec) com partículas de madeira de eucalipto tratadas com 4,1\% de PE-g-MA, nas composições de 40/60\%; 50/50 e 60/40\% em massa, analisadas a $20^{\circ} \mathrm{C} / \mathrm{min}$, sob atmosfera inerte de nitrogênio. 
Tabela 1. Análise termogravimétrica do homopolímero LDPErec e dos compósitos PEuc/LDPErec, contendo partículas sem tratamento e tratadas com PE-gMA (Temperatura inicial de degradação).

\begin{tabular}{|c|c|c|c|c|c|c|c|}
\hline \multirow[t]{3}{*}{ Amostras } & \multirow{3}{*}{$\begin{array}{c}\begin{array}{c}\text { LDPErec } \\
\text { puro }\end{array} \\
\text { TG }\left({ }^{\circ} \mathbf{C}\right) \\
\end{array}$} & \multicolumn{6}{|c|}{ PEuc/LDPErec } \\
\hline & & \multicolumn{2}{|c|}{ Sem tratamento } & \multicolumn{2}{|c|}{ 4,1\% PE-g-MA } & \multicolumn{2}{|c|}{ 8,8\% PE-g-MA } \\
\hline & & $\mathbf{T G}^{1}\left({ }^{\circ} \mathrm{C}\right)$ & $\mathbf{T G}^{2}\left({ }^{\circ} \mathbf{C}\right)$ & $\mathbf{T G}^{1}\left({ }^{\circ} \mathrm{C}\right)$ & $\mathbf{T G}^{2}\left({ }^{\circ} \mathbf{C}\right)$ & $\mathbf{T G}^{1}\left({ }^{\circ} \mathbf{C}\right)$ & $\mathbf{T G}^{2}\left({ }^{\circ} \mathbf{C}\right)$ \\
\hline $0 / 100$ & 225 & - & - & - & - & - & - \\
\hline $40 / 60$ & - & 268 & 450 & 275 & 446 & 273 & 458 \\
\hline $50 / 50$ & - & 244 & 458 & 264 & 462 & 283 & 446 \\
\hline $60 / 40$ & - & 260 & 454 & 275 & 457 & 272 & 455 \\
\hline
\end{tabular}

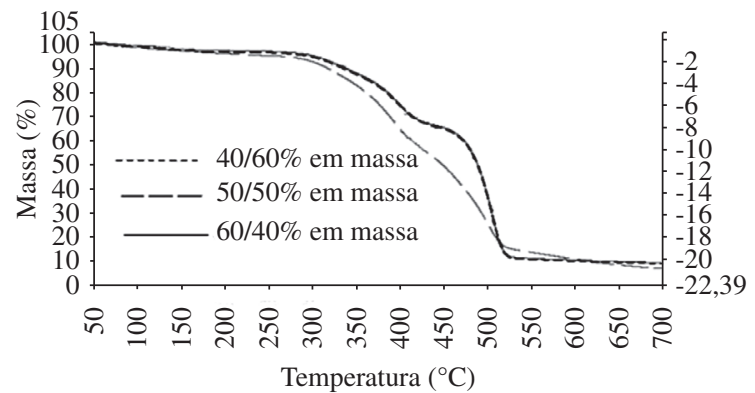

Figura 6. Curvas termogravimétricas dos compósitos (PEuc/LDPErec) com partículas de madeira de eucalipto tratadas com $8,8 \%$ de PE-g-MA, nas composições de $40 / 60 \%$; 50/50 e 60/40\% em massa, analisadas a $20^{\circ} \mathrm{C} / \mathrm{min}$, sob atmosfera inerte de nitrogênio.

A presença do PE-g-MA como agente compatibilizante, acarretou em um ligeiro aumento das temperaturas iniciais de degradação térmica da fase rica em partículas de madeira de eucalipto $\left(\mathrm{TG}^{1}\right)$ com o aumento da proporção de partículas de madeira nos compósitos. Esse comportamento comprova a atuação do PE-g-MA como agente compatibilizante entre essas partículas e o LDPE reciclado, aumentando a interação entre esses dois materiais. Porém esse efeito compatibilizante exercido pelo PE-g-MA foi inferior ao efeito causado pela heterogeneidade do sistema, quando se aumenta a proporção de partículas nos compósitos, podendo-se concluir que as composições desses materiais apresentam um limite máximo de atuação das partículas de madeira de eucalipto como carga e/ou reforço.

A Figura 7 mostra o gráfico contendo o comportamento de temperatura inicial de degradação térmica desses compósitos.

\section{Comportamento mecânico dos compósitos de PEuc/LDPErec}

A Tabela 2 apresenta todos os valores do comportamento mecânico do LDPErec puro e dos compósitos de PEuc/LDPErec.

Através do ensaio de tração pode-se fazer uma comparação entre os valores de LDPErec puro de resistência à tração no escoamento (21,1 $\mathrm{MPa} \pm 0,5)$ e na ruptura $(18,0 \mathrm{MPa} \pm 1,8)$, e o módulo de flexão $(1.457 \mathrm{MPa} \pm 191,2)$ e os valores de resistência à tração no escoamento e na ruptura dos compósitos de partículas tratadas e sem tratamento.

Pelos resultados apresentados nessa tabela pode-se verificar que houve um aumento dos valores de resistência à tração para todas as composições dos compósitos obtidos com partículas tratadas, quando comparados aos valores das partículas sem tratamento, fazendo com que os valores de re-

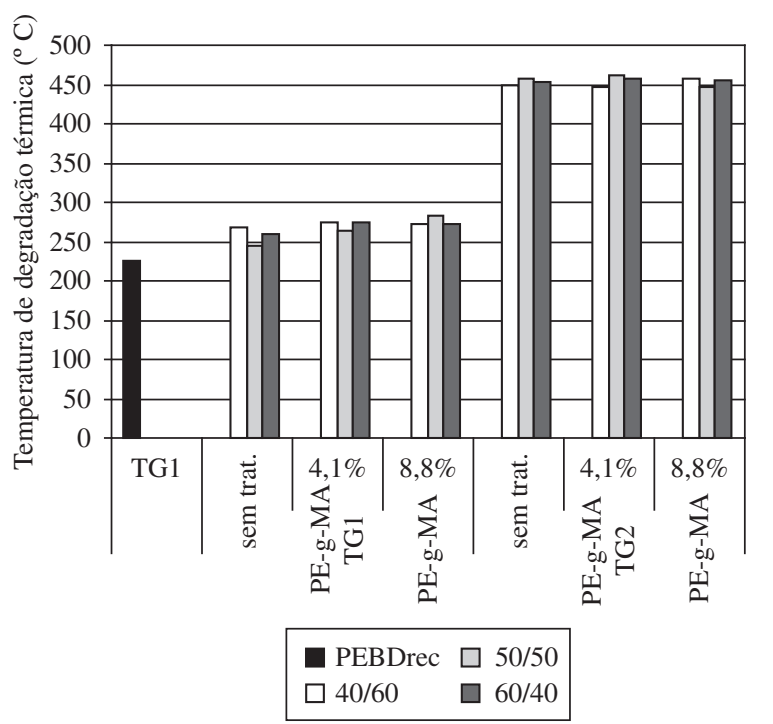

Figura 7. Comportamento de degradação térmica (TG) do homopolímero LDPErec e dos compósitos de PEuc/LDPErec.

sistência à tração desses compósitos se aproximem do valor de resistência à tração do LDPErec puro. Esse resultado pode estar implicado ao fato do PE-g-MA estar atuando como agente compatibilizante entre as partículas de madeira e o LDPErec, fazendo com que essas partículas de eucalipto dispersas fiquem mais aderidas à matriz de polímero reciclado. Esse comportamento relaciona a presença de grupamentos anidrido maleico no PE-g-MA que auxilia na interação entre as cadeias de LDPErec e celulose, contribuindo para uma melhor adesão entre a carga e a matriz polimérica. Observa-se também que o compósito de composição 50/50\%, em massa, contendo partículas de eucalipto tratadas com 8,8\% PE-g-MA apresenta os valores de resistência à tração no escoamento e na ruptura superiores ao valor do LDPErec puro, mostrando que o efeito de agente compatibilizante exercido pelo PE-g-MA complementa a ação de reforço exercida pelas partículas de madeira, nessa composição, superando a heterogeneidade do sistema e proporcionando melhor interação entre as partículas de madeira e a matriz polimérica.

No entanto, o aumento da proporção de partículas de madeira de eucalipto para $60 \%$, em massa, acarretou na diminuição dos valores de resistência à tração dos compósitos de PEuc/LDPErec, para partículas tratadas. Nesse caso, a maior quantidade de partículas de eucalipto sofre maior influência da heterogeneidade do sistema do que pela presença do agente compatibilizante. 
Tabela 2. Comportamento mecânico do LDPErec puro e dos compósitos de PEuc/LDPErec.

\begin{tabular}{|c|c|c|c|}
\hline Amostra & $\begin{array}{l}\text { Resistência à tração no } \\
\text { escoamento (MPa) }\end{array}$ & $\begin{array}{l}\text { Resistência à tração na } \\
\text { ruptura (MPa) }\end{array}$ & Módulo de flexão (MPa) \\
\hline LDPErec $100 \%$ & $21,1 \pm 0,5$ & $18,0 \pm 1,8$ & $1.457 \pm 191,2$ \\
\hline \multicolumn{4}{|c|}{ Compósitos de PEuc/LDPErec } \\
\hline \multirow[t]{2}{*}{ Amostras } & \multicolumn{3}{|c|}{ Resistência à tração no escoamento (MPa) } \\
\hline & Sem tratamento & 4,1\% PE-g-MA & 8,8\% PE-g-MA \\
\hline $40 / 60 \%$ & $11,2 \pm 1,5$ & $18,6 \pm 1,4$ & $19,0 \pm 2,7$ \\
\hline $50 / 50 \%$ & $9,0 \pm 1,3$ & $18,1 \pm 1,4$ & $23,7 \pm 4,4$ \\
\hline \multirow[t]{3}{*}{$60 / 40 \%$} & $10,5 \pm 1,9$ & $15,6 \pm 2,6$ & $15,9 \pm 3,3$ \\
\hline & \multicolumn{3}{|c|}{ Resistência à tração na ruptura (MPa) } \\
\hline & Sem tratamento & 4,1\% PE-g-MA & 8,8\% PE-g-MA \\
\hline $40 / 60 \%$ & $3,4 \pm 0,3$ & $14,9 \pm 6,2$ & $11,9 \pm 4,1$ \\
\hline $50 / 50 \%$ & $3,2 \pm 0,3$ & $14,8 \pm 6,9$ & $23,2 \pm 4,3$ \\
\hline \multirow[t]{3}{*}{$60 / 40 \%$} & $6,7 \pm 4,9$ & $14,9 \pm 2,9$ & $15,4 \pm 3,8$ \\
\hline & \multicolumn{3}{|c|}{ Módulo de flexão (MPa) } \\
\hline & Sem tratamento & 4,1\% PE-g-MA & 8,8\% PE-g-MA \\
\hline $40 / 60 \%$ & $3.173 \pm 519,1$ & $3.022,4 \pm 673,7$ & $3.152,6 \pm 369,6$ \\
\hline $50 / 50 \%$ & $3.989,6 \pm 341,5$ & $2.872,0 \pm 228,4$ & $4.054,0 \pm 490,1$ \\
\hline $60 / 40 \%$ & $3.528 \pm 305,8$ & $5.260,8 \pm 370,8$ & $3.770,7 \pm 322,9$ \\
\hline
\end{tabular}

A variação das concentrações do agente compatibilizante não proporcionou alterações significativas dos valores de resistência à tração para os compósitos. Segundo Lu et al. (2005) e Liang et al. (2003) $)^{[17,18]}$, existe um valor ótimo de concentração desses agentes para promover uma melhor adesão entre a matriz e a fase dispersa e conseqüentemente, para que os compósitos apresentem melhoria em suas propriedades de mecânicas. Provavelmente a concentração de PE-g-MA de 8,8\%, em massa, está acima da concentração indicada para promover melhorias significativas na interação entre a matriz e a fase dispersa desses materiais.

Os valores de resistência à tração na ruptura, levando-se em consideração os respectivos desvios-padrão, mostram que esses materiais compósitos não apresentaram melhorias significativas nessa propriedade, quando comparados com o valor de resistência à tração na ruptura do LDPErec. Porém, comparando-se os valores de resistência à tração na ruptura dos compósitos obtidos com partículas de madeira de eucalipto não tratadas e tratadas com o agente compatibilizante, pode-se concluir que as partículas de eucalipto não tratadas apresentam uma fraca aderência à matriz de LDPErec, acarretando em valores de resistência à tração na ruptura bem menores que o valor apresentado por LDPErec puro.

Quando tratadas com PE-g-MA, nas duas concentrações pesquisadas (4,1 e 8,8\%, em massa), as partículas de eucalipto, os valores de resistência à tração na ruptura dos compósitos de PEuc/LDPErec aumentaram, ficando próximos ao valor verificado pelo LDPErec puro, dependendo da composição. Esse comportamento mostra a ação de reforço dessas partículas de madeira tratadas para os materiais compósitos, mesmo levando em consideração a heterogeneidade desses sistemas.

Também foi possível observar pelos valores de módulo de flexão do homopolímero LDPErec puro e dos compósitos de PEuc/LDPErec que houve, em média, um aumento de todos os valores das amostras de compósitos de PEuc/LDPErec, dependendo do teor de partículas de eucalipto presentes. Como esperado, observações similares fo- ram reportadas por Arbelaiz et al (2005) ${ }^{[19]}$ onde o módulo de flexão de compósitos de madeira aumenta com o aumento do teor da fibra, mostrando a atuação das partículas de madeira como reforço para a matriz polimérica. O ligeiro decréscimo do valor de módulo de flexão apresentado pelo compósito de composição $(60 / 40 \%)$ provavelmente é influenciado pela composição das misturas. O aumento da quantidade de partículas de madeira leva à diminuição da homogeneidade entre a matriz e a fase dispersa, acarretando em uma diminuição das propriedades mecânicas desse material, sem a interferência do agente compatibilizante.

\section{Microscopia Eletrônica de Varredura (SEM)}

A microscopia eletrônica de varredura confirma o comportamento mecânico, a partir da análise morfológica de alguns compósitos de PEuc/LDPErec, contendo partículas de madeira de eucalipto sem tratamento e tratadas com PE-g-MA (Figuras 8 e 9).

Foi visualizado pelas micrografias dos compósitos de PEuc/LDPErec de composições 40/60 e 50/50\%, em massa, contendo partículas sem tratamento e tratadas com 4,1 e $8,8 \%$ de PE-g-MA (Figuras 8 e 9) que os compósitos contendo partículas tratadas proporcionaram maior encobrimento dessas partículas de madeira, diferenciando da morfologia dos compósitos obtidos com partículas sem tratamento, comprovando que a ausência do agente compatibilizante causou uma pobre adesão interfacial entre a matriz polimérica e fase dispersa.

\section{Absorção de Água}

A Figura 10 mostra o comportamento de absorção de água dos compósitos de PEuc/LDPErec, de acordo com o teor de partículas e de agente compatibilizante.

Pode-se verificar pelos resultados que a variação da composição dos compósitos influencia na absorção de água. Os compósitos de composição $60 / 40 \%$, em massa absorvem 
maior quantidade de água, devido à presença de maior quantidade de partículas de madeira. O caráter polar da celulose faz com esses materiais apresentem maior afinidade pela água. No entanto, os compósitos com partículas de madeira tratadas com PE-g-MA absorveram menor quantidade de água, quando comparados com compósitos com partículas sem tratamento. As composições de 40/60 e 50/50\% dos compósitos com partículas tratadas com 4,1 e 8,8\% de PE-g-MA não apresentaram variações significativas na absorção de água. No entanto, a composição de 60/40\%, com partículas tratadas com $8,8 \%$ de PE-g-MA, apresentou uma diminuição na absorção de água após 24 horas de análise. Nesse caso, a estrutura química PE-g-MA teve uma maior influência, recobrindo melhor as partículas de madeira de eucalipto, acarretando em uma menor absorção de água.

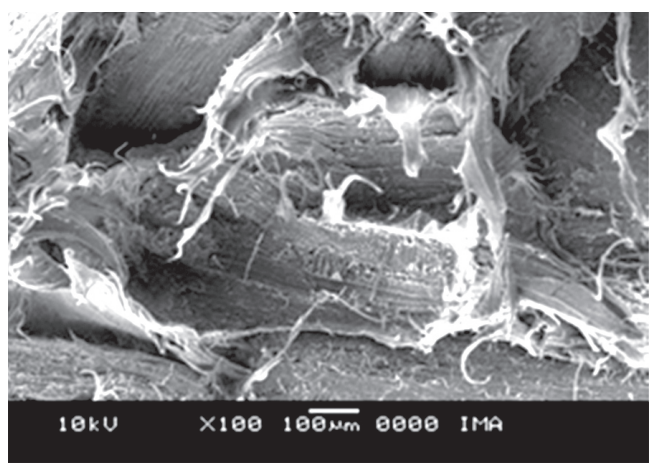

(a)

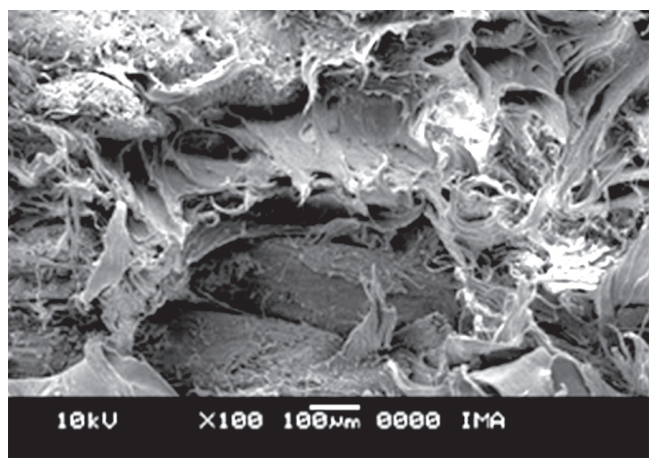

(b)

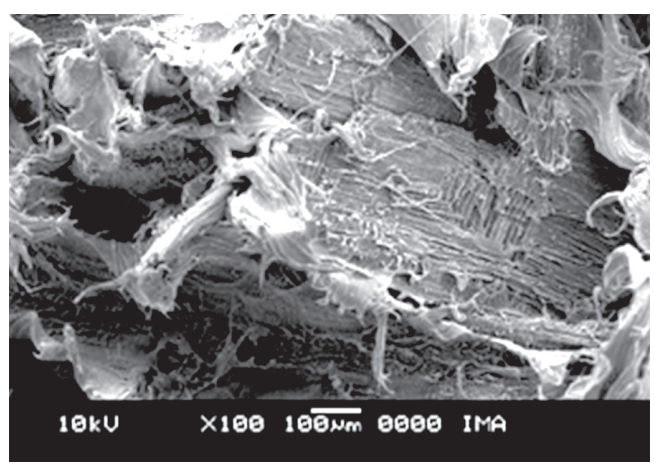

(c)

Figura 8. Micrografias dos compósitos (PEuc/LDPErec) de composição $40 / 60 \%$, a) partículas sem tratamento; b) partículas tratadas com $4,1 \%$ de PE-g-MA; e c) Partículas tratadas com $8,8 \%$ de PE-g-MA.

\section{Conclusões}

Foi possível observar que o PE-g-MA atuou como agente compatibilizante entre a matriz e a carga desses materiais, provocando uma pequena melhora nas propriedades térmicas e mecânicas dos compósitos de PEuc/LDPErec, quando comparados com compósitos contendo partículas não tratadas. Além disso, a presença do PE-g-MA fez com que as partículas de madeira de eucalipto absorvessem menor quantidade de água, podendo concluir que a estrutura química do PE-g-MA teve uma maior influência, recobrindo melhor as partículas de madeira, quando comparadas com as partículas sem tratamento. As micrografias comprovam a melhoria da adesão interfacial entre os componentes do compósito (PEuc/LDPErec), quando utiliza-se o PE-g-MA como agente compatibilizante, corroborando com os resul-

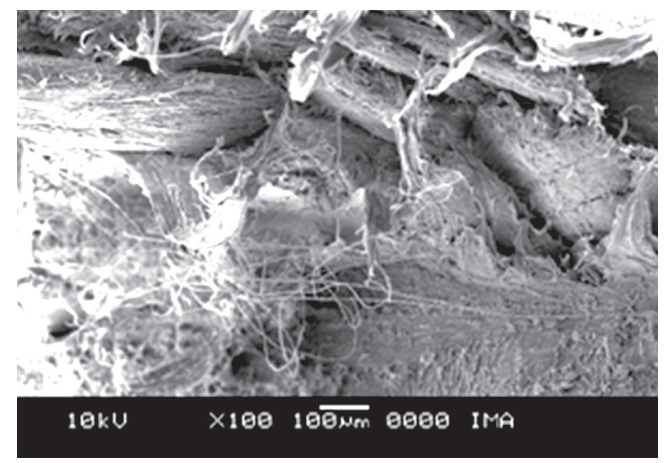

(a)

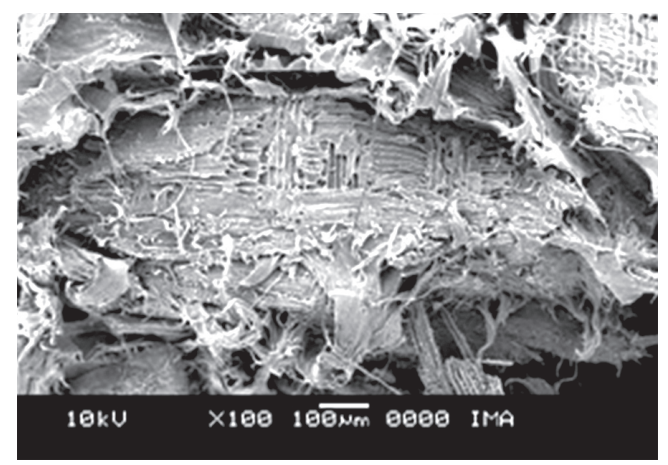

(b)

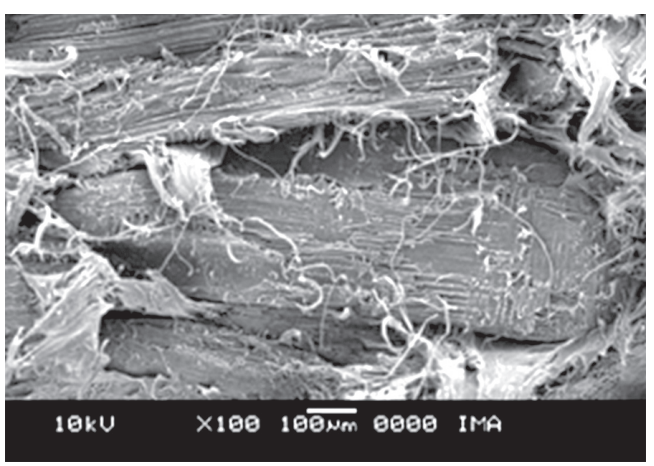

(c)

Figura 9. Micrografias dos compósitos (PEuc/LDPErec) de composição $50 / 50 \%$, a) partículas sem tratamento; b) partículas tratadas com $4,1 \%$ de PE-g-MA; e c) Partículas tratadas com 8,8\% de PE-g-MA. 
tados obtidos nos ensaios mecânicos. Desse modo, a produção de um novo material a partir da utilização de polietileno reciclado reforçado com madeira de reflorestamento é perfeitamente viável, em termos de melhoria de algumas propriedades.

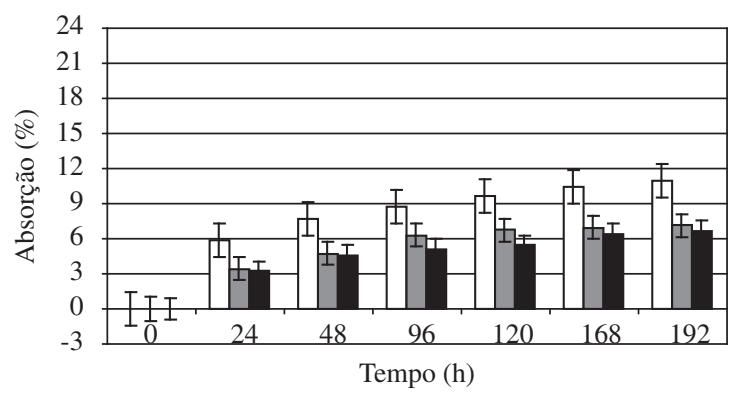

$\square$ 40/60 0\% PE-g-MA $\square$ 40/60 4,1\% PE-g-MA — 40/60 8.8\% PE-g-MA

(a)

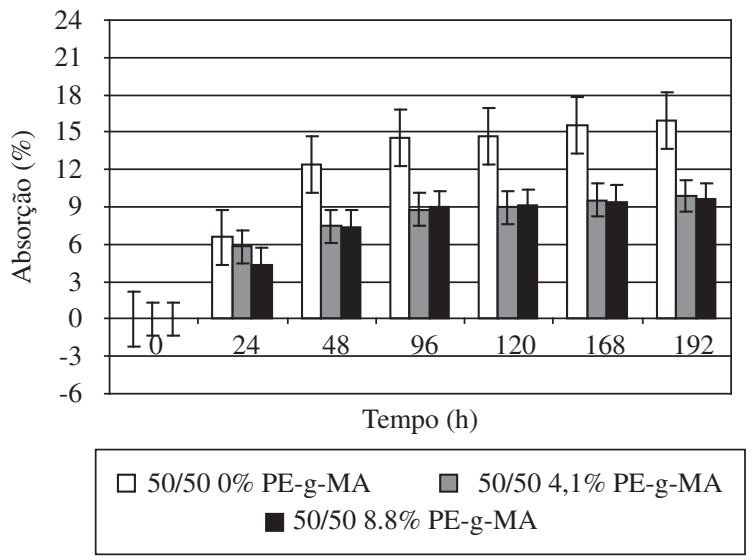

(b)

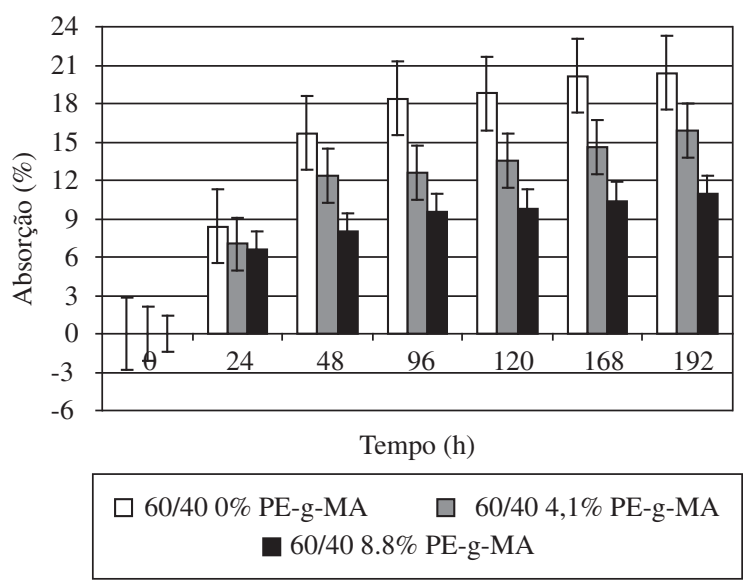

(c)

Figura 10. Absorção de água em função do tempo de imersão para compósitos: a) $40 / 60 \%$; b) $50 / 50 \%$; e c) $60 / 40 \% \mathrm{~m} / \mathrm{m}$, com as partículas de madeira de eucalipto sem tratamento e tratadas.

\section{Referências Bibliográficas}

1. Martins, F. A.; Suarez, J. C. M. \& Mano, E. B. - Polímeros: Ciência e Tecnologia, out/dez, p.27 (1999).

2. Paiva, J. M. F \& Frollini, E. - "Natural fibers reinforced thermoset composites", in: Natural Polymers and Agrofibers based Composites, E. Frollini, A. Leão, L. H. C. Mattoso (ed), Embrapa Instrumentação Agropecuária, S.Carlos, SP (2000).

3. Paiva, J. M. F; Silva, S. P.; Tanaka, I. A.; Trindade, W. G.; Angelucci, C. A. \& Frollini, E. - "Impact Strength of phenolic matrices reinforced with lignocellulosic material" in: Natural Polymers and Composites, L. H. C. Mattoso, E. Frollini, A. Leão (ed), p.460 (2000).

4. Yamaji, F. M. \& Bonduelle, A. - Revista Floresta, 34, jan/abr, p.59 (2004).

5. Selkea, S. E. \& Wichman, I. - Composites: Part A, 35, p.321 (2004).

6. Callister, W. D. Jr. - "Ciência e Engenharia de Materiais: Uma Introdução", Rio de Janeiro, LTC (2002).

7. Costa, T. H. S.; Carvalho, D. L.; Souza, D. C. S.; Coutinho, F. M. B.; Pinto, J. C. \& Kokta, B. V. - Polymer Testing, 19, p.419 (2000).

8. Coutinho, F. M. B.; Costa, T. H. S.; Carvalho, D. L.; Gorelova, M. M. \& De Santa Maria, L. C. - Polymer Testing, 17, p.299 (1998).

9. Coutinho, F. M. B. \& Costa, T. H. S. - Polymer Testing, 18, p.581 (1999).

10. Ichazo, M. N.; Albano, C.; González, J.; Perera, R. \& Candal, M. V. - Composite Structures, 54, p.207 (2001).

11. Kazayawoko, M.; Balatinecz, J. J. \& Matuana, L. M. - J. Mat. Sci., 34, p.6189 (1999).

12. Wu, J.; Yu, D.; Chi-Ming, C.; Kim, J. \& Mai, Y. - J. Appl. Polym. Sci., 76, p.1000 (2000).

13. Bohn, A. R. \& Szücs, C. A. - "Influência da espessura dos anéis de crescimento no comportamento mecânico dos elementos de madeira laminada colada", in: Anais do Encontro Brasileiro em Madeiras e em Estruturas de Madeira - EEUFMG; CEFET; IBRAMEN, p.579, Belo Horizonte (1995).

14. Iwakiri, S.; Cunha, A. B.; Albuquerque, C. E. C.; Gorniak, E. \& Mendes, L. M. - Scientia Agraria, 1, 1-2, p.23 (2000).

15. American Society for Testing and Materials - ASTM, D 638-03, Standard Test Method for Tensile Properties of Plastics, 2003.

16. American Society for Testing and Materials - ASTM D 790-03 Standard Test Methods for Flexural Properties of Unreinforced and Reinforced Plastics and Electrical Insulating Materials, 2003.

17. Lu, J. Z.; Wu, Q. \& Negulescu, I. I.; - J. Appl. Polym. Sci., 96, p.93 (2005).

18. Liang, G.; Xu, J.; Bao, S. \& Xu, W. - J. Appl. Polym. Sci., 91, p.3974 (2004).

19. Arbelaiz, A.; Fernández, B.; Ramos, J. A.; Retegi, A.; Llano-Ponte, R. \& Mondragon, I. - Composites Science and Technology, 65, p.1582 (2005).

Enviado: $18 / 04 / 07$ Reenviado: $14 / 08 / 07$

Aceito: 14/09/07 